\title{
The National Diabetic Retinopathy Laser Treatment Audit. II. Proliferative retinopathy
}

\begin{abstract}
The National Diabetic Retinopathy Laser Treatment Audit is a prospective survey of laser treatment for diabetic retinopathy throughout the United Kingdom. This paper presents data on 284 patients who were undergoing their first panretinal photocoagulation for proliferative retinopathy during a 2 month period in 1995, describing the demographic features, the level of systematic screening, the sources of referral, and the waiting times. For those cases where proliferative retinopathy was present at the first ophthalmology outpatient visit, the retinopathy was detected as a result of systematic screening in $\mathbf{4 6 . 8 \%}$, whilst $\mathbf{2 8 . 7 \%}$ presented symptomatically. Of these patients $\mathbf{2 8 . 4} \%$ waited for more than 12 weeks from referral to the time of laser treatment, but once the patient had been listed for laser treatment this was performed within 8 weeks in $95.3 \%$. The retinopathy features and the type of treatment given are also described. Compared with the DRS and ETDRS recommendations, at least $32.5-40.2 \%$ of eyes may be undertreated initially, and for those with highrisk characteristics these figures were at least 30.8-38.5\%.
\end{abstract}

Key words Diabetic retinopathy, Proliferative retinopathy, Laser, Panretinal photocoagulation, Process, Screening

The effectiveness of photocoagulation in the treatment of proliferative diabetic retinopathy has been demonstrated in a number of studies. ${ }^{1-4}$ Despite the availability of an effective treatment many patients do still go blind from diabetic retinopathy, and it remains a leading cause of blindness in patients of working age in the Western world. ${ }^{5-8}$ The Saint Vincent Declaration, endorsed by the British Diabetic Association and the Department of Health, set as one of its targets reducing blindness due to diabetes by one-third or more. ${ }^{9}$
C.C. BAILEY, J.M. SPARROW,

R.H.B. GREY, H. CHENG
Whether this can be achieved depends on a number of factors including screening and the efficacy of treatment.

This national audit was designed to examine the processes of delivery of photocoagulation treatment for diabetic retinopathy throughout the United Kingdom. This paper presents data on patients with proliferative retinopathy.

\section{Methods}

The full description of the methodology for this audit is described elsewhere. ${ }^{10}$ In brief, all consultant ophthalmologists in the UK who undertake laser treatment for diabetic retinopathy were invited to participate. Eligible for inclusion were those patients with proliferative diabetic retinopathy undergoing panretinal photocoagulation for the first time in a particular eye during June and July 1995. The ophthalmologist performing the laser treatment was asked to complete a confidentially coded questionnaire containing questions on retinopathy features, visual acuity prior to photocoagulation, grade of ophthalmologist performing the treatment, and the treatment given. The questionnaire also asked for the sources of referral, the mode of detection of the retinopathy, and waiting times for the clinic and laser treatment.

For those patients who were undergoing bilateral simultaneous treatment $(n=20)$, the eye with the worse visual acuity was included in the audit. Where the vision was equal in the two eyes, the right or left eye was randomly chosen for further analysis.

\section{Results}

\section{Patient characteristics}

Two hundred and eighty-four patients undergoing the first panretinal

photocoagulation in one eye were recruited into the audit. Their mean age was 54.8 years, range $17-85$ years; $42.1 \%$ were female, and $57.9 \%$ were

\author{
C.C. Bailey \\ J.M. Sparrow \\ R.H.B. Grey \\ $H$. Cheng \\ The Royal College of \\ Ophthalmologists \\ 17 Cornwall Terrace \\ London NW1 4QW, UK
}

The Department of Health provided funding for this project. 
Table 1. Source of referral to the ophthalmology clinic

\begin{tabular}{lcc}
\hline & $n$ & $\%$ \\
\hline Hospital physician & 116 & 41.3 \\
General practitioner & 79 & 28.1 \\
Optometrist & 37 & 13.2 \\
Hospital ophthalmologist & 37 & 13.2 \\
Self-referral & 10 & 3.6 \\
Ophthalmic medical practitioner & 2 & 0.7 \\
Total & 281 & 100 \\
\hline
\end{tabular}

male (chi-squared $=6.96, p<0.01$ ). The right eye was recruited into the audit in $54.6 \%$ of patients, and the left eye was recruited in $45.4 \%$.

Diabetes mellitus was diagnosed before the age of 30 years and required insulin treatment (younger-onset) in $31.2 \%(64)$ of these patients. Diabetes mellitus was diagnosed after the age of 30 years (older-onset) in $68.8 \%$ (141). Of the older-onset group, $43.3 \%$ (61) required insulin treatment, $50.3 \%$ (71) required oral hypoglycaemic agents, and $6.4 \%$ (9) were controlled with diet alone.

The mean duration of diagnosed diabetes for these cases with proliferative retinopathy was 16.8 years (range $0.1-54.9$ years). For those with younger-onset diabetes mellitus, the mean duration of diabetes was 24.7 years (range 7.3-54.9 years). For the older-onset group, the mean duration of diagnosed diabetes was 13.1 (range 0.1-34.1 years).

\section{Sources of referral and level of systematic screening}

The sources of referral to the ophthalmology clinic are shown in Table 1. A total of $69.4 \%$ of patients were referred by their hospital physician or general practitioner, while in $13.2 \%$ of cases the referral was initiated by the optometrist. Proliferative retinopathy was present at the first ophthalmology clinic appointment in $35.9 \%(94)$, and for this group (excluding self-referrals) the presence of proliferative retinopathy was correctly identified by the referrer in $48.2 \%$ of cases.

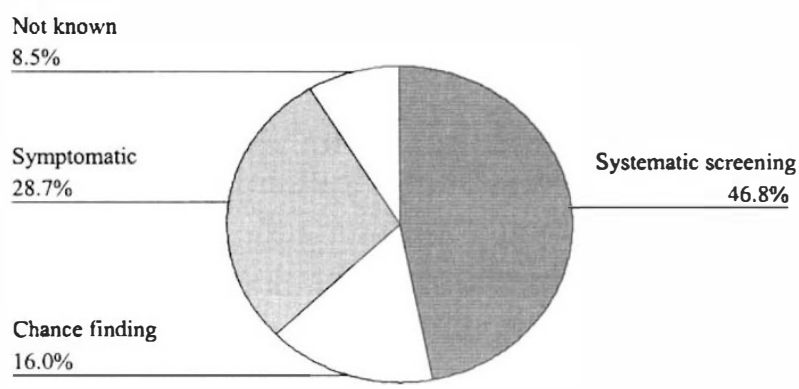

Fig. 1. Mode of detection of the retinopathy that led to referral to the ophthalmology outpatient clinic, for those patients in whom proliferative retinopathy was present at the first ophthalmology outpatient visit $(\mathrm{n}=94)$. In this context, systematic screening refers to the detection of retinopathy as a result of at least annual, planned, dilated fundal examination by an optometrist or doctor. A chance finding refers to the detection of the pathology in a patient who did not undergo systematic screening as defined above, and who did not present symptomatically.
Table 2. Retinopathy features $(n=284)$

\begin{tabular}{lcc}
\hline Retinopathy feature present & $n$ & $\%$ \\
\hline NVD & 144 & 50.7 \\
NVE & 169 & 59.5 \\
Concurrent maculopathy & 86 & 30.3 \\
Previously treated maculopathy & 76 & 26.7 \\
Vitreous haemorrhage & 73 & 25.7 \\
Rubeosis & 10 & 3.5 \\
Traction & 16 & 5.6 \\
Traction retinal detachment & 2 & 0.7 \\
\hline
\end{tabular}

NVD, new vessels on the disc; NVE, new vessels elsewhere.

For all the cases undergoing panretinal photocoagulation, the detection of retinopathy that led to referral to the ophthalmology clinic was the result of systematic screening in $55.3 \%$. In this context, systematic screening was defined as at least annual, dilated fundal examination by an optometrist or doctor. This group also included those patients who had maculopathy detected or treated prior to the development of proliferative retinopathy, and was not therefore a true reflection of the detection rate from screening for preproliferative or proliferative retinopathy per se. Fig. 1 is a truer reflection of the mode of detection of retinopathy for those in whom proliferative retinopathy was present at the first ophthalmology outpatient visit. For this group, $28.7 \%$ presented symptomatically, and the retinopathy was detected by systematic screening in $46.8 \%$.

\section{Retinopathy features present}

The retinopathy features are described in Table 2. Patients who had new vessels on the disc (NVD) comprised $50.7 \%$, whilst $59.5 \%$ had new vessels elsewhere (NVE). Overall $61.2 \%$ of patients had either NVD and/or NVE associated with vitreous haemorrhage, broadly equivalent to the 'high-risk characteristics' as defined by the Diabetic Retinopathy Study. ${ }^{2}$

In the fellow eye, $53.1 \%$ had proliferative retinopathy, $38.5 \%$ had maculopathy, $27.3 \%$ had background retinopathy only, and $1.1 \%$ had no diabetic retinopathy.

Table 3. Best-recorded visual acuity prior to treatment

\begin{tabular}{lccccc}
\hline & \multicolumn{2}{c}{$\begin{array}{c}\text { Visual acuity in the } \\
\text { eye to be treated }\end{array}$} & & \multicolumn{2}{c}{$\begin{array}{c}\text { Visual acuity in } \\
\text { the better eye }\end{array}$} \\
\cline { 2 - 3 } \cline { 5 - 6 } Visual acuity & $n$ & $\%$ & & $n$ & $\%$ \\
\hline $6 / 5$ & 32 & 11.3 & & 43 & 15.2 \\
$6 / 6$ & 52 & 18.4 & & 68 & 24.0 \\
$6 / 9$ & 73 & 25.7 & & 87 & 30.7 \\
$6 / 12$ & 46 & 16.2 & & 37 & 13.1 \\
$6 / 18$ & 28 & 9.9 & & 26 & 9.2 \\
$6 / 24$ & 15 & 5.3 & & 5 & 1.8 \\
$6 / 36$ & 10 & 3.5 & & 8 & 2.8 \\
$6 / 60$ & 6 & 2.1 & & 4 & 1.4 \\
$<6 / 60$ & 21 & 7.4 & & 5 & 1.8 \\
Total & 283 & 100 & & 283 & 100 \\
\hline
\end{tabular}

${ }^{\text {a }}$ The best-recorded visual acuity using spectacles and/or pinhole if required. 
Table 4. Waiting times to be seen in the ophthalmology clinic and for the laser treatment

\begin{tabular}{|c|c|c|c|}
\hline Wait (weeks) & $n$ & $\%$ & Cumulative \% \\
\hline \multicolumn{4}{|l|}{ For laser treatment } \\
\hline Treatment on same day & 52 & 18.9 & 18.9 \\
\hline $1-4$ weeks & 166 & 60.4 & 79.3 \\
\hline $5-8$ & 44 & 16.0 & 95.3 \\
\hline $9-12$ & 11 & 4.0 & 99.3 \\
\hline$>12$ & 2 & 0.7 & 100 \\
\hline Total & 275 & 100 & \\
\hline \multicolumn{4}{|l|}{ For clinic appointment ${ }^{a}$} \\
\hline Up to 4 & 41 & 48.8 & 48.8 \\
\hline $5-8$ & 23 & 27.4 & 76.2 \\
\hline $9-12$ & 11 & 13.1 & 89.3 \\
\hline $13-16$ & 5 & 5.9 & 95.2 \\
\hline$>16$ & 4 & 4.8 & 100 \\
\hline Total & 84 & 100 & \\
\hline \multicolumn{4}{|l|}{ Overall $^{\text {b }}$} \\
\hline Up to 4 & 24 & 29.6 & 29.6 \\
\hline $5-8$ & 21 & 26.0 & 55.6 \\
\hline $9-12$ & 13 & 16.0 & 71.6 \\
\hline $13-16$ & 11 & 13.6 & 85.2 \\
\hline$>16$ & 12 & 14.8 & 100 \\
\hline Total & 81 & 100 & \\
\hline
\end{tabular}

${ }^{a}$ Refers to cases where proliferative retinopathy was present at the first ophthalmology outpatient visit.

${ }^{b}$ Refers to the overall wait from the time of referral to the laser treatment for those cases where proliferative retinopathy was present at the first ophthalmology outpatient visit.

\section{Best-recorded visual acuity in the eye to be treated}

The best-recorded visual acuity in the eye prior to treatment is shown in Table 3, which reveals that $55.4 \%$ of eyes to be treated had a best-recorded visual acuity of $6 / 9$ or better, whilst $18.3 \%$ had a best-recorded visual acuity of $6 / 24$ or worse. Where the visual acuity was less than $6 / 9$ in the treated eye, $24.2 \%$ had some degree of cataract sufficient to cause a reduction in visual acuity. In the better eye, $70.0 \%$ of patients had a visual acuity of $6 / 9$ or better, whilst $7.8 \%$ had a visual acuity of $6 / 24$ or worse.

Waiting times to be seen in the ophthalmology outpatient clinic and for the laser treatment

The waiting times to be seen in the ophthalmology clinic and for the laser treatment are shown in Table 4. The median wait for the laser treatment from listing was 2 weeks (range $0-14$ weeks), $79.3 \%$ being treated within 4 weeks whilst $4.7 \%$ waited for more than 8 weeks from listing for their laser treatment. For those patients in whom proliferative retinopathy was present at the first ophthalmology outpatient visit, the median wait for the clinic appointment was 5 weeks (range 0-30 weeks), $48.8 \%$ being seen within 4 weeks of referral, while $10.7 \%$ waited for more than 12 weeks for their clinic appointment. For these cases, the overall wait from the time of referral to the time of laser treatment was a median 7 weeks (range $0-31$ weeks), and $28.4 \%$ waited for more than 12 weeks from the time of referral.
Table 5. Grade of ophthalmologist performing the panretinal photocoagulation

\begin{tabular}{lrc}
\hline Grade of ophthalmologist & $n$ & $\%$ \\
\hline Consultant & 100 & 35.6 \\
Associate specialist & 35 & 12.5 \\
Staff grade & 30 & 10.7 \\
Clinical assistant & 19 & 6.8 \\
Fellow & 4 & 1.4 \\
Senior registrar & 31 & 11.0 \\
Registrar & 44 & 15.7 \\
Senior house officer & 10 & 3.6 \\
SHO or other under supervision & 8 & 2.8 \\
Total & 281 & 100 \\
\hline
\end{tabular}

\section{Grade of ophthalmologist performing panretinal photocoagulation}

The grade of ophthalmologist performing panretinal photocoagulation is shown in Table 5. A consultant ophthalmologist performed $35.6 \%$ of the photocoagulation.

\section{Treatment given}

In $41.2 \%$ of cases it was considered that the ophthalmologist intended to give the initial panretinal photocoagulation in one sitting. This was based upon the statement that the next appointment was for reassessment rather than specifically for further treatment. In $55.6 \%$ of cases it was intended to divide the initial treatment into more than one session, whilst in $3.2 \%$ of cases this information was not recorded. The spot sizes used for the panretinal photocoagulation are shown in Table 6 and the number of burns given at the first treatment is shown in Table 7. For the group in which the intention was to give the initial panretinal photocoagulation treatment in one session ( $n=117)$, the median number of burns given at the first session was 1146 (range 325-2686). 3.4\% of eyes were given fewer than 500 burns, and $18.8 \%$ of eyes were given fewer than 800 burns.

A peri- or retrobulbar injection of anaesthetic was given in $6.9 \%$ of all patients undergoing panretinal photocoagulation, and in $5.7 \%$ of those who were given the initial treatment in one session.

Table 6. Spot size used for the laser treatment ${ }^{\mathrm{a}}$

\begin{tabular}{lrr}
\hline Spot size used $(\mu \mathrm{m})$ & $n$ & $\%$ \\
\hline 100 & 2 & 0.7 \\
150 & 5 & 1.8 \\
200 & 108 & 38.3 \\
250 & 10 & 3.5 \\
300 & 19 & 6.7 \\
350 & 1 & 0.4 \\
400 & 29 & 10.3 \\
500 & 107 & 37.9 \\
600 & 1 & 0.4 \\
Total & 282 & 100 \\
\hline
\end{tabular}

${ }^{a}$ Does not necessarily represent the size of the laser spot on the retina, in view of the range of possible lenses used for the treatment. Refers to the most frequently used spot size for the treatment. 
Cases (\%) for whom intention was to give PRP in one session

\begin{tabular}{lc} 
No. of burns & $\begin{array}{c}\text { in one session } \\
(n=117)\end{array}$ \\
\hline$<500$ & 3.4 \\
$500-999$ & 26.5 \\
$1000-1499$ & 41.0 \\
$1500-1999$ & 17.1 \\
$2000-2500$ & 10.3 \\
$>2500$ & 1.7 \\
\hline
\end{tabular}

Cases (\%) for whom intention was to give PRP in more than one session $(n=158)$

9.5
44.9
31.7
11.4
2.5
0

All cases (\%) $(n=284)$
All cases $(\%)$
Cases (\%) with NVD and/or NVE associated with vitreous haemorrhage, , for whom the intention was to give PRP in

$\begin{array}{rc}7.4 & 0 \\ 36.3 & 24.6 \\ 35.2 & 47.7 \\ 14.4 & 20.0 \\ 5.6 & 7.7 \\ 1.1 & 0\end{array}$

${ }^{a}$ Broadly equivalent to the high-risk characteristics as defined by the DRS.

\section{Treatment for concurrent maculopathy}

For those patients in whom maculopathy was present concurrently with proliferative retinopathy $(n=86)$, $26.7 \%$ (23) had undergone treatment for the maculopathy prior to the panretinal photocoagulation, whilst $45.3 \%$ (39) had the maculopathy treated at the same session. In $22.1 \%$ of cases (19) the maculopathy was to be treated after the panretinal photocoagulation, whilst in 5.8\% (5) there was no specific intention to treat the maculopathy. For those in whom treatment of the maculopathy was to be after the panretinal photocoagulation, this was intended to be within 1 month of the panretinal photocoagulation in $10.5 \%$, within 1-2 months in $31.6 \%$ and had not been decided in $57.9 \%$. For those whose concurrent maculopathy was treated prior to panretinal photocoagulation, this was performed between 0.4 and 109 weeks before, with a median value of 18 weeks.

\section{Follow-up}

When the next appointment was specifically for further treatment $(n=158)$, the median interval was 2 weeks (range 1-11 weeks). Further treatment was to be given within 2 weeks in $60.3 \%$, within $3-4$ weeks in $28.8 \%$, whilst $8.3 \%$ were to be brought back for further treatment after 5-6 weeks and $2.6 \%$ were to be brought back for further treatment after 6 weeks. When the next appointment was for reassessment rather than specifically for further treatment $(n=117)$, the median follow-up was 6 weeks (range 1-13 weeks).

Reassessment was to be within 4 weeks in $30.2 \%$, within $5-8$ weeks in $55.1 \%$, whilst $14.7 \%$ were to be brought back for reassessment within 9-13 weeks.

\section{Discussion}

\section{Patient characteristics}

The sample had significantly more men than women. The Wisconsin Epidemiologic Study of Diabetic Retinopathy (WESDR) showed a significant association between male gender and the development of proliferative retinopathy in those patients in whom diabetes was diagnosed before the age of 30 years and required insulin treatment, where diabetes had been present for more than 10 years, ${ }^{11}$ but this was not found for those patients with older-onset diabetes. ${ }^{12}$ The excess of male patients with proliferative retinopathy probably reflects the fact that diabetes is now more common in men than women in the UK. The 1993 Health Survey of England ${ }^{13}$ reported a prevalence of diabetes of $3 \%$ in men compared with $2 \%$ in women, and in the UK Prospective Diabetes Study the ratio of men to women was 3:2. ${ }^{14}$

In this audit, $32 \%$ of the patients with proliferative retinopathy had diabetes of younger onset (diagnosed before the age of 30 years and requiring insulin treatment). This may be compared with the estimate that $10-25 \%$ of adults with diabetes in the UK have type 1 diabetes.

The mean duration of diabetes from diagnosis to the time of recruitment into the audit was lower for the older-onset than the younger-onset groups. For those with younger-onset diabetes, $1.8 \%$ had a duration of disease of less than 10 years, with a mean of 24.7 years. For the older-onset group, $40.7 \%$ had had diabetes for less than 10 years with a mean duration from diagnosis of 13.2 years. This is in accordance with expectations based upon the WESDR, which showed that during the first 9 years of diabetes the rates of panretinal photocoagulation treatment were higher in older-onset compared with younger-onset patients, ${ }^{15}$ and in the UK Prospective Diabetes Study diabetic retinopathy was present at the time of diagnosis in at least $18 \%$ of patients with type 2 diabetes. ${ }^{16}$ These observations probably reflect the fact that type 2 diabetes may have been present for several years before diagnosis.

The number of patients treated for new proliferative retinopathy during the study period can be extrapolated to represent about 2260 patients annually, which takes into account a level of participation of just over $75 \%$ as indicated by the validation exercise. ${ }^{10}$ The 1993 Health Survey for England estimated that there are 1380000 adults with diabetes in the UK. ${ }^{13}$ Thus it would appear that about $0.16 \%$ of adults with diabetes in the UK develop proliferative retinopathy in one eye that is detected and treated each year. The WESDR estimated the incidence of progression to proliferative retinopathy to be $2.7 \%$ per year for the first 4 years for those patients with younger-onset diabetes, whilst this was $2.0 \%$ for the older-onset group on insulin treatment, and $0.6 \%$ for the older-onset group not requiring insulin. ${ }^{17}$ In the UK, 
the incidence of sight-threatening retinopathy is thought to be $1.2-1.5 \% .{ }^{18,19} \mathrm{~A}$ comparison of the number of patients expected to undergo first panretinal photocoagulation treatment per annum in the UK and the numbers actually treated would suggest that there is a significant amount of sight-threatening retinopathy that is not currently being detected or treated.

\section{Level of systematic screening}

For those patients in whom proliferative retinopathy was present at the first outpatient clinic, the retinopathy was detected by systematic screening in $46.8 \%$, and $28.7 \%$ of patients presented symptomatically. This large percentage of patients presenting symptomatically suggests that a significant amount of proliferative disease is not being detected or treated. The presence of the proliferative retinopathy was correctly diagnosed by the referrer in only $48.2 \%$ of cases compared with $80.8 \%$ for maculopathy, when the disease was noted at first presentation to the ophthalmology clinic. ${ }^{10}$ Moreover, $64.8 \%$ of maculopathy was detected by systematic screening, and $13.5 \%$ presented symptomatically. It would therefore appear that primary screening is less efficient at detecting proliferative retinopathy than maculopathy. Since the detection of proliferative retinopathy relies on screening in the asymptomatic stage, the sensitivity of primary screening needs to improve.

\section{Waiting times}

While the median wait for treatment was only 2 weeks and $79.3 \%$ of patients were treated within 4 weeks of listing, $23.8 \%$ of patients with established disease still had to wait for more than 8 weeks to be seen in the outpatient clinic and the overall wait for treatment from referral was more than 12 weeks in $28.4 \%$. Improvement of the waiting time to be seen in the ophthalmology clinic is indicated.

\section{Treatment given at the first session}

The Diabetic Retinopathy Study (DRS) protocol for panretinal photocoagulation recommended 800-1600 argon laser burns of $500 \mu \mathrm{m}$ spot size extending to or beyond the vortex ampullae for eyes with high-risk characteristics. ${ }^{2}$ The ETDRS protocol for full scatter treatment was developed from this and recommended $1200-1600$ argon laser burns of $500 \mu \mathrm{m}$ spot size. ${ }^{20}$ The ETDRS recommended that the treatment should be performed in two or more episodes, which were no more than 2 weeks apart, and that no more than 900 burns were to be applied in one session.

An estimate of the area of retina ablated can be described using the standard formula of $\pi r^{2}$ multiplied by the number of burns (Fig. 2). Fig. 3 shows the results of this calculation for eyes with NVD and/or NVD associated with vitreous haemorrhage (broadly equivalent to the 'high-risk characteristics' as defined by the DRS). To avoid complexity the type of lens used in treatment was not specified in the questionnaire.

However, since the quadraspheric lens gives the greatest possible magnification of the commonly available contact lenses used in treatment (by a factor of 1.9), one can assume a range of magnification of the spot size of $1: 1$ to 1:1.9 when calculating the areas of treated retina.

For the group in whom the intention was to give the initial panretinal photocoagulation in one session $(n=117)$, the median retinal area treated was $98.2 \mathrm{~mm}^{2}$

$120-$

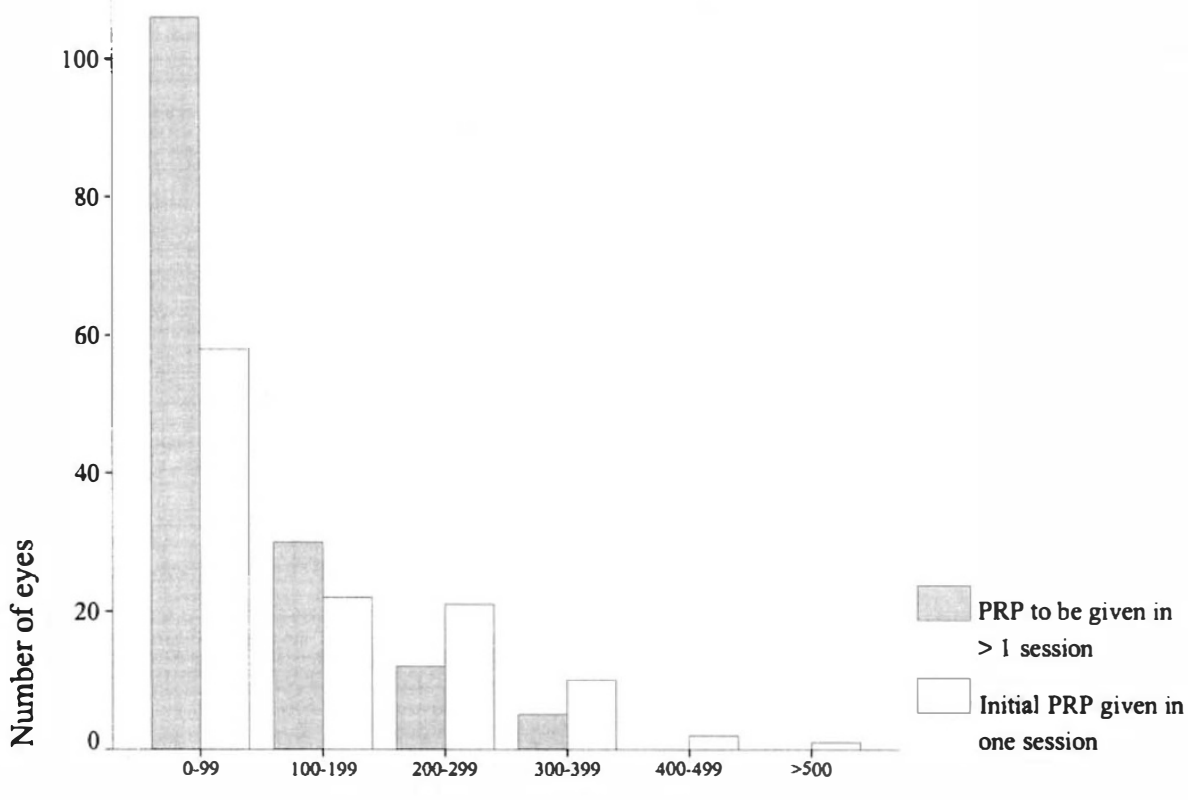

Retinal area (square $\mathrm{mm}$ )

Fig. 2. Estimate of the area of retina ablated at the first treatment session. 


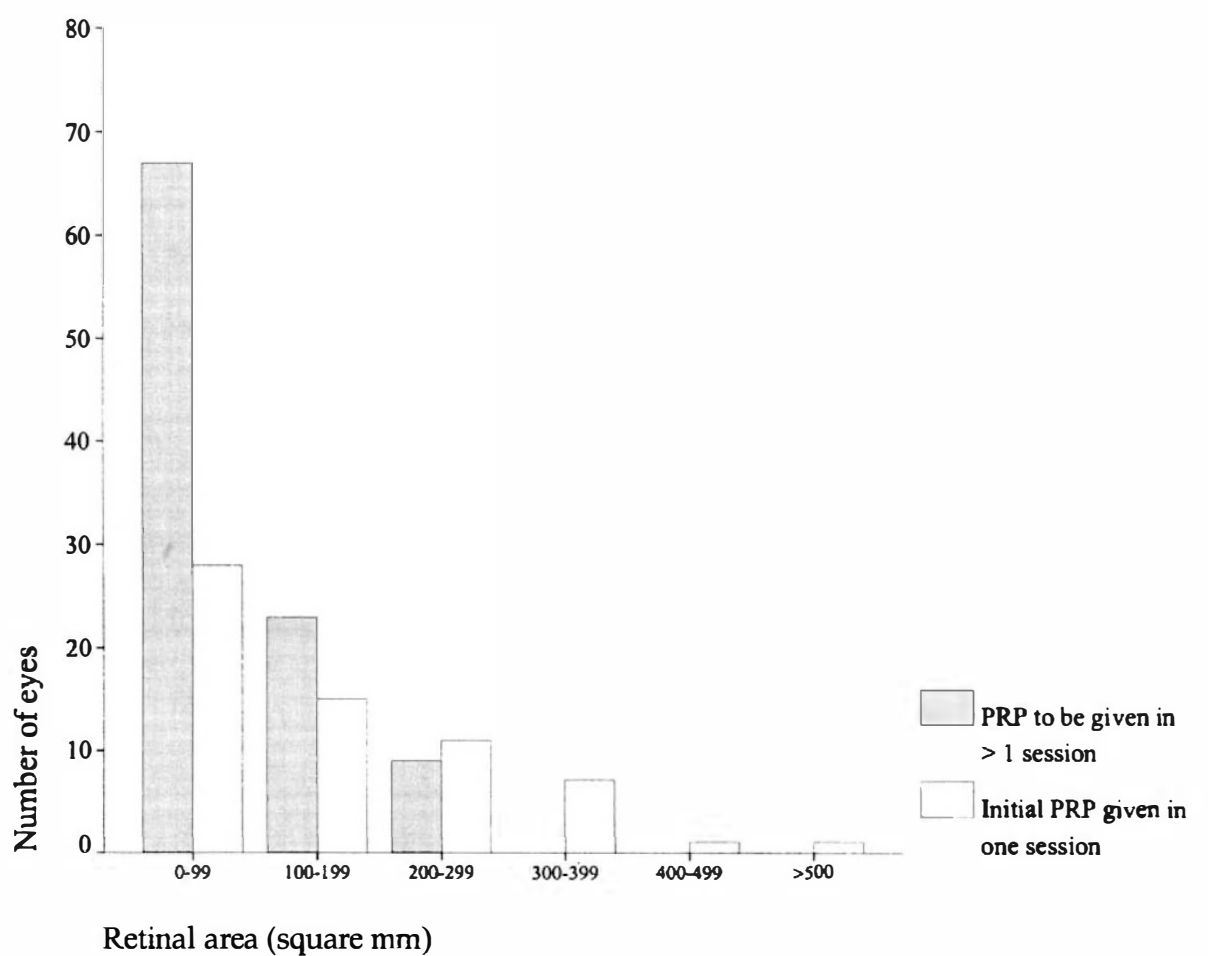

Fig. 3. Estimate of the area of retina ablated for eyes with new vessels on the disc and/or new vessels elsewhere associated with vitreous haemorrhage (broadly equivalent to 'high-risk characteristics' as defined by the DRS).

(range $6.7-682.5 \mathrm{~mm}^{2}$ ), or $354.4 \mathrm{~mm}^{2}$ (range 24.3-2464 $\mathrm{mm}^{2}$ ) based upon a calculation assuming use of the quadraspheric lens for all cases. For the subgroup with the equivalent of high-risk characteristics who were to be given their initial panretinal photocoagulation in one session $(n=65)$, the median retinal area treated was $104.6 \mathrm{~mm}^{2}$ (range $10.4-682.5 \mathrm{~mm}^{2}$ ), or $377.6 \mathrm{~mm}^{2}$ (range $37.6-2464 \mathrm{~mm}^{2}$ ) if the quadraspheric lens had been used for all cases. According to the DRS protocol, the recommended amount of retina treated is equivalent to an area of 157 to $314 \mathrm{~mm}^{2}$, whilst the lower limit for the full panretinal photocoagulation treatment as recommended by the ETDRS is equivalent to an area of $236 \mathrm{~mm}^{2}$. In this study, for those eyes in which the intention was to give the initial panretinal photocoagulation in one sitting, $64.1 \%$ of eyes received less than the lower limit of the DRS protocol, and $81.2 \%$ received less than the lower limit of the ETDRS protocol. For the subgroup with the equivalent of high-risk characteristics who were given their initial treatment in one session $(n=65)$, the corresponding figures were $60 \%$ and $80 \%$ respectively. If a spot magnification factor of 1.9 is taken for all cases, then $32.5 \%$ of eyes received less than the lower limit of the DRS protocol, and $40.2 \%$ of eyes received less than the lower limit of the ETDRS protocol. For the subgroup with the equivalent of highrisk characteristics who were treated in one sitting these results were $30.8 \%$ and $38.5 \%$ respectively.

These smaller areas of retinal ablation would appear to result more from the spot size used than from the number of burns given, since only $18 \%$ of eyes that received the initial panretinal photocoagulation treatment in one session were given fewer than 800 burns. Hulbert and Vernon ${ }^{21}$ reported their practice of using $200 \mu \mathrm{m}$ size burns in the initial treatment protocol, since this was often easier for the patient to tolerate and for the operator to deliver. Moreover, they found that this had beneficial effects on the ability of patients to retain driving fields. They found that between 3000 and 3500 burns induced regression in all but the most severe cases. To ablate the same area of retina as the equivalent of 800 burns of $500 \mu \mathrm{m}$ spot size would require 4997 burns of $200 \mu \mathrm{m}$ spot size. In this audit for those patients treated with burns of $200 \mu \mathrm{m}$ spot size, in whom the intention was to give the initial treatment in one session, the mean number of burns given was 1190, and for those with high-risk characteristics in this category the mean number of burns was $1127(n=23)$. For those in whom a $500 \mu \mathrm{m}$ spot size was used and the intention was to give the initial treatment in one session, the mean number of burns used was 1301, and was 1315 for those with highrisk characteristics. It would therefore appear that the number of burns given was not increased for those cases in which a smaller spot size was used, although it is possible that a more magnifying lens was used for those eyes in which a smaller spot size was recorded. For eyes with the equivalent of high-risk characteristics $(n=172)$, the most frequently used spot size was $200 \mu \mathrm{m}$ in $41.2 \%$ of eyes, whilst this was $500 \mu \mathrm{m}$ in $32.4 \%$ of eyes. It would not appear that the smaller spot size is being reserved specifically for lower-risk eyes in order to try to preserve field. It is possible that the DRS and ETDRS recommend more treatment than is actually needed in many cases, but in practice some eyes do require more treatment than described by these studies. ${ }^{22,23}$ There therefore appears to be a tendency to undertreat initially, compared with 
the DRS and ETDRS protocols. The follow-up study will show whether this is limited to the initial stages and whether outcome is affected.

It is interesting to note that for these patients undergoing the first panretinal photocoagulation for proliferative retinopathy in one eye, proliferative retinopathy and/or maculopathy was present in the other eye in $71.6 \%$ of cases.

\section{Presence and treatment of concurrent maculopathy}

In the DRS, by a combination of clinical examination and fluorescein angiography, $15.2 \%$ of eyes had definite macular oedema, and a further $29.2 \%$ had questionable macular oedema. ${ }^{24}$ In this audit, concurrent maculopathy was said to be present in $30.3 \%$, and maculopathy that had been previously treated and had resolved was present in $26.7 \%$. The ETDRS compared giving immediate panretinal photocoagulation with either simultaneous or delayed focal treatment, and found that the former strategy was associated with the least visual loss. ${ }^{4}$ The DRS also discussed the timing of treatment for concurrent maculopathy and suggested that 'perhaps eliminating or reducing retinal thickening by applying focal photocoagulation for macular edema before beginning scatter treatment might reduce the risk of visual loss' ${ }^{24}$ Some ophthalmologists make an exception to this approach, especially for young patients with IDDM who have aggressive peripheral ischaemia associated with macular oedema that may resolve following panretinal photocoagulation. ${ }^{25}$ In this audit, concurrent maculopathy was treated before or at the same session as the panretinal photocoagulation in $72 \%$, whilst in $22 \%$ it was intended to treat the maculopathy after the panretinal photocoagulation. In the latter group, the mean age was 56.9 years (range 31-81 years), and these patients would not generally appear to fit into the category of young patients with aggressive disease in whom it may be best to perform panretinal photocoagulation first.

In conclusion, this audit has provided unique data concerning the processes of delivery of photocoagulation treatment for proliferative retinopathy throughout the United Kingdom. The issues that need to be addressed are:

1. The long waiting time for a clinic appointment for cases with established disease. This is an organisational issue that needs to be tackled locally.

2. The large proportion of eyes presenting symptomatically $(28.7 \%)$, which suggests poor screening procedures.

3. The possible initial undertreatment of some eyes with proliferative retinopathy. The follow-up study will show whether this is limited to the initial stages and whether outcome is affected.

We would like to thank all the ophthalmologists who took part in this audit, and made this study possible. We would also like to thank Professor D. McLeod, Mr J. S. Shilling and Mr J. Talbot for their input as members of the steering committee for this audit, Professor A. R. Rosenthal as chairman of the audit committee, and the audit secretary Mrs Janice Samson for her efficient assistance.

\section{References}

1. British Multicentre Photocoagulation Trial. Proliferative diabetic retinopathy: treatment with xenon-arc photocoagulation. Interim report of multicentre randomised controlled trial. BMJ 1977;I:739-41.

2. Diabetic Retinopathy Study Research Group. Photocoagulation treatment of proliferative diabetic retinopathy. Clinical application of diabetic retinopathy study findings: DRS report no. 8. Ophthalmology 1981;88:583-600.

3. Hercules BL, Gayed II, Lucas SB, Jeacock J. Peripheral retinal ablation in the treatment of proliferative diabetic retinopathy: a three-year interim report of a randomised, controlled study using the argon laser. Br J Ophthalmol 1977;61:555-63.

4. Early Treatment Diabetic Retinopathy Study Research Group. Early photocoagulation for diabetic retinopathy: ETDRS report no. 9. Ophthalmology 1991;98:766-85.

5. Evans J, Rooney C, Ashwood F, Dattani N, Wormald R. Blindness and partial sight in England and Wales: April 1990-March 1991. Health Trends 1996;28:5-12.

6. Ghafour IM, Allan D, Foulds WS. Common causes of blindness and visual handicap in the west of Scotland. Br J Ophthalmol 1983;67:209-13.

7. Klein R, Klein BEK, Moss SE. Visual impairment in diabetes. Ophthalmology 1984;91:1-9.

8. Moss SE, Klein R, Klein BE. Ten-year incidence of visual loss in a diabetic population. Ophthalmology 1994;101:1061-70.

9. Diabetes care and research in Europe: The Saint Vincent Declaration. Diabet Med 1990;7:360.

10. Bailey CC, Sparrow JM, Grey RHB, Cheng H. The National Diabetic Retinopathy Laser Treatment Audit. I. Maculopathy. Eye 1998;12:69-76.

11. Klein R, Klein BEK, Moss SE, Davis MD, DeMets DL. The Wisconsin Epidemiologic Study of Diabetic Retinopathy. II. Prevalence and risk of diabetic retinopathy when age at diagnosis is less than 30 years. Arch Ophthalmol 1984;102:520-6.

12. Klein R, Klein BEK, Moss SE, Davis MD, DeMets DL. The Wisconsin Epidemiologic Study of Diabetic Retinopathy. III. Prevalence and risk of diabetic retinopathy when age at diagnosis is 30 or more years. Ophthalmology 1984;102:527-32.

13. Bennett N, Dodd T, Flatley, J, Freeth S, Bolling K. Health survey for England 1995. Social Survey Division of the Office of Population Censuses and Surveys, London: HMSO, 1995.

14. UK Prospective Diabetes Study. IV. Characteristic of newly presenting type 2 diabetic patients: male preponderance and obesity at different ages. Diabet Med 1988;5:154-9.

15. Klein R, Klein BEK, Moss SE, Davis MD, DeMets DL. The Wisconsin Epidemiologic Study of Diabetic Retinopathy. VI. Retinal photocoagulation. Ophthalmology 1987;94:747-53.

16. Aldington SJ, Stratton IS, Kohner EM, Matthews DR, Turner RC. Prevalence of retinopathy at diagnosis of type 2 diabetes in the UK Prospective Diabetes Study. Diabet Med 1994;11:S43-4.

17. Klein R, Klein BE, Moss SE, Cruickshanks KJ. The Wisconsin Epidemiologic Study of Diabetic Retinopathy. XIV. Ten-year incidence and progression of diabetic retinopathy. Arch Ophthalmol 1994;112:1217-28.

18. Foulds WS, McCuish A, Barrie T, et al. Diabetic retinopathy in the west of Scotland: its detection and prevalence, and the cost-effectiveness of a proposed screening programme. Health Bull 1983;41:318-26. 
19. Brunel University Health Economics Research Group. Diabetic retinopathy screening project: Special Medical Development Project Team. Health Economics Research Group report no. 8, 1990.

20. Early Treatment Diabetic Retinopathy Study Research Group. Techniques for scatter and local photocoagulation treatment of diabetic retinopathy: ETDRS report no. 3. Int Ophthalmol Clin 1987;27:254-64.

21. Hulbert MF, Vernon SA. Passing the DVLC field regulations following bilateral pan-retinal photocoagulation in diabetics. Eye 1992;6:456-60.
22. Aylward GW, Pearson RV, Jagger JD, Hamilton AM. Extensive argon laser photocoagulation in the treatment of proliferative diabetic retinopathy. $\mathrm{Br} \mathrm{J}$ Ophthalmol 1989;73:197-201.

23. Reddy VM, Zamora RL, Olk RJ. Quantitation of retinal ablation on proliferative diabetic retinopathy. Am J Ophthalmol 1995;119:760-6.

24. Diabetic Retinopathy Study Research Group. Macular edema in Diabetic Retinopathy Study patients. Ophthalmology 1987;94:754-60.

25. Hamilton AMP, Ulbig MW, Polkinghorne P. Management of diabetic retinopathy. London: BMJ Publishing Group, 1996. 